\title{
Inferior colliculus of the house mouse
}

\author{
III. Response probabilities and thresholds of single units \\ to synthesized mouse calls compared to tone and noise bursts
}

\author{
Günter Ehret and Anne J.M. Moffat* \\ Fakultät für Biologie, Universität Konstanz, Postfach 5560, D-7750 Konstanz, Federal Republic of Germany
}

Accepted February 11, 1985

Summary. 1. The response probabilities and thresholds of single units in the IC of the house mouse to tones, white noise, and four synthesized mouse calls have been measured in animals anesthetized with either sodium pentobarbital or chlorprothixene.

2. According to their responsiveness tested within an intensity range up to $80 \mathrm{~dB}$ SPL neurons were classified as follows: tone insensitive, tone preferred, call-model insensitive, call-model preferred (to various degrees), noise insensitive, and noise preferred.

3. In the DC+DM of the IC significantly higher proportions of neurons were noise insensitive compared with the $\mathrm{CN}$ of the IC (Table 1).

4. DC+DM units from pentobarbital anesthetized animals were significantly less responsive to two of the mouse calls and generally showed a higher selectivity in response to all four calls compared with units from the $\mathrm{CN}$. These differences were not seen in chlorprothixene anesthetized mice (Figs. 2, 4).

5. Sodium pentobarbital significantly increased the noise response thresholds in both $\mathrm{DC}+\mathrm{DM}$ and $\mathrm{CN}$ (Figs. 3, 5).

6 . The response selectivity to mouse calls correlated significantly with the shape and the sharpness of excitatory tuning curves (Table 2, Fig. 7).

7. Results are discussed with regard to anesthetic effects, differences in selectivity in the DC+

Abbreviations: $C F$ characteristic frequency; $C N$ central nucleus of the inferior colliculus; $D C$ dorsal cortex of the inferior colliculus; $D M$ dorsomedial nucleus of the inferior colliculus; $I C$ inferior colliculus; $N$-group mice anesthetized with sodium pentobarbital; T-group mice anesthetized with chlorprothixene; $S P L$ sound pressure level

* Present address: Department of Biology, University of Oregon, Eugene, OR 97403, USA
DM compared to the $\mathrm{CN}$ and in comparison with studies on other animals.

\section{Introduction}

In the ascending auditory pathway, single unit responses to broadband stimuli such as noise or animal calls become less predictable with increasing order of neural centers. While auditory nerve and ventral cochlear nucleus units all show excitation to stimulation with broadband noise or more complex spectra if the spectra have components within the area of the excitatory tuning curve (Møller 1970; Ruggero 1973; Kiang and Moxon 1974; Rhode et al. 1978; Ehret and Moffat 1984), this is not the case in all higher centers. Already in the dorsal cochlear nucleus, there are neurons that are not excited by broadband noise even though their characteristic frequencies (CFs) are within the noise spectrum (Young and Brownell 1976). In the medial geniculate body (Symmes et al. 1980) and auditory cortex (e.g. Newman 1979; Langner et al. 1981) the selectivity of unit responses to complex spectra increases substantially and response probabilities and thresholds to complex stimuli can often not be predicted by even a detailed knowledge of tone responses.

There are only limited data indicating the degree of response selectivity to complex spectra at the auditory midbrain level (Scheich et al. 1977; Engelstätter et al. 1980; Ehret and Moffat 1985). We know of only one study (Suga 1969) in which units of the IC were quantified according to their response selectivities to tone and noise bursts. The degree of response specialization to complex and 
wideband calls of an animal's repertoire is known only for the guinea fowl (Scheich et al. 1977). The available data suggest that response probabilities and thresholds of auditory midbrain units to complex sounds are not necessarily related to response probabilities and thresholds for tonal stimuli.

In the present study we want to extend our knowledge of response specialization in the auditory pathway, which is essential for the understanding of sound discrimination and recognition in animals, by providing basic quantitative data on: (a) the proportions of units in the IC of the mouse showing various degrees of selectivity to tones, broadband noise and synthesized mouse calls, (b) the relationships between tone, noise and call response thresholds, (c) the relationships between call response selectivity and shapes and sharpness of excitatory tuning curves to tones. Neurons were identified as being from the central nucleus $(\mathrm{CN})$ or the dorsal cortex plus dorsomedial nucleus $(\mathrm{DC}+\mathrm{DM})$. In addition, we used two anesthetics, sodium pentobarbital to make our data comparable with those of earlier studies (Suga 1969; Engelstätter et al. 1980) and chlorprothixene (Taractan), which is believed to yield results very similar to those from unanesthetized mice (Dräger 1975).

\section{Materials and methods}

Preparation. House mice (Mus musculus, outbred strain NMRI, aged 2-4 months) were used. Surgical, stimulus, recording and data analysis procedures have been described in detail in an earlier report (Ehret and Moffat 1985). Fourtyone mice were anesthesized with sodium pentobarbital $(80 \mathrm{mg} / \mathrm{kg})$ for surgery and in 21 animals the anesthetic level was maintained by further injection of pentobarbital $(35 \mathrm{mg} / \mathrm{kg})$ when necessary. These mice are referred as the $\mathrm{N}$-group. In the other 20 animals a light anesthetic state was maintained by injections of chlorprothixene (Taractan; $3.5 \mathrm{mg} / \mathrm{kg}$ ) supplemented in 11 animals by small dosages of pentobarbital $(10 \mathrm{mg} / \mathrm{kg})$ about every $3 \mathrm{~h}$. Animals with chlorprothixene anesthesia will be referred as the T-group.

Animals were rigidly fixed in a headholder and placed on a feedback controlled heating pad (rectal temperature always $37 \pm 1^{\circ} \mathrm{C}$ ) in a sound-proof and anechoic room. The skull was opened and the dura over the IC was removed. Steel electrodes were stereotactically positioned into the same central IC area as described in the accompanying study (Ehret and Moffat 1985) and were advanced by a remote controlled microdrive. Heart and breathing rates were monitored. Recordings were only made under stable physiological conditions.

Sound stimuli. Sounds were presented free-field either through a dynamic speaker (Transco HTF 80/5) or through an electrostatic speaker (Machmerth et al. 1975). The frequency response of the dynamic speaker was flat within $\pm 4 \mathrm{~dB}$ between 1 and $20 \mathrm{kHz}$, while that of the electrostatic speaker was flat within $\pm 2 \mathrm{~dB}$ between 15 and $100 \mathrm{kHz}$ and showed a decrease of the frequency response below $15 \mathrm{kHz}$ of nearly $9 \mathrm{~dB}$ /octave. Pure tones were produced in a function generator (Wavetek 132), shaped into bursts of $300 \mathrm{~ms}$ flat top duration plus $10 \mathrm{~ms}$ rise and fall times and $300 \mathrm{~ms}$ inter-burst intervals, and passed through an attenuator (Hewlett-Packard 350D) to an electronic adder. White noise was produced in a random noise generator (General Radio 1390-B), passed through two digital bandpass filters in series (Rockland 852; initial slopes $96 \mathrm{~dB} /$ octave), through an electronic switch for burst shaping as described for the tone bursts, and through an attenuator (Hewlett-Packard 350D) to the adder. The following models of intraspecific calls were synthesized: Model A was a filtered white noise (bandpass $40-60 \mathrm{kHz}, 96 \mathrm{~dB}$ /octave initial slopes), which had been shown to be a behaviorally effective facsimile of mouse pup ultrasonic calls (Ehret and Haack 1982). Model B was a filtered harmonic series (fundamental frequency $4 \mathrm{kHz}$, high cutoff at $12 \mathrm{kHz}$ with $48 \mathrm{~dB} /$ octave slope) imitating the distress call of adult mice (Ehret 1975). Model C was a harmonic series with $5 \mathrm{kHz}$ fundamental frequency extending up to $80 \mathrm{kHz}$, imitating the distress call of young mouse pups (Ehret 1975). Model D consisted basically of model B plus a band of filtered white noise (bandwidth $25-50 \mathrm{kHz}$, slopes $48 \mathrm{~dB} /$ octave). The $4-12 \mathrm{kHz}$ harmonic series and the noise band were equalized in intensity. This sound approximates the defensive call of nonreceptive females in sexual encounters (Ehret 1975). The call models were generated with function generators (Exact 129), the previously mentioned noise generator, attenuators, filters, pulse shapers, adder and amplifiers (Hewlett-Packard 466A and Exact 170). Durations and repetition rates were adapted to the respective natural calls (model $\mathrm{A}$; duration $80+5 \mathrm{~ms}$ rise and fall times, repetition rate 3 calls/s; models $B, C, D$ : duration $100+10 \mathrm{~ms}$ rise and fall times, repetition rate $1 \mathrm{call} / \mathrm{s})$. To calibrate the sounds used, all stimuli were recorded by a calibrated microphone (Brüel and Kjaer 4135) at the position of the animal's pinna. The sound pressure level (SPL) was determined with use of a measuring amplifier (Brüel and Kjaer 2606) and the spectrum was verified by a spectrum analyzer (Nicolet $466 \mathrm{~A}$ ). Figure 1 shows the spectra of the call models used as recorded at the animal's pinna.

Procedure. When a single unit was encountered, its position in the IC was noted. Then its threshold to tone bursts at its characteristic frequency $(\mathrm{CF})$ and to bursts of bandpassed noise (either $1.5-20 \mathrm{kHz}$, delivered by the dynamic speaker for units with CFs up to $17.5 \mathrm{kHz}$, or $15-80 \mathrm{kHz}$ delivered by the electrostatic speaker for units with CFs higher than $17.5 \mathrm{kHz}$ ) were determined audiovisually. The call models were then presented in the sequence $\mathrm{A}-\mathrm{D}$, and thresholds determined when possible. SPLs of up to $80 \mathrm{~dB}$ total SPL (re $20 \mu \mathrm{Pa}$ ) could be presented without distortion. Units which were classified as 'insensitive' did not show any excitatory response (tonic, phasic, phasictonic, pauser, long latency, or 'off') to any SPL up to $80 \mathrm{~dB}$. Finally, excitatory frequency tuning curves were obtained.

\section{Results}

\section{Response probabilities}

We studied 101 neurons in the N-group and 63 in the T-group. Table 1 summarizes the distribution of neurons in the different response classes. In both anesthetic groups some neurons did not respond with excitation either to tones (tone insensitive) or to any call model (call model insensitive) or to broadband noise (noise insensitive). Other neurons had excitatory responses only to tones 

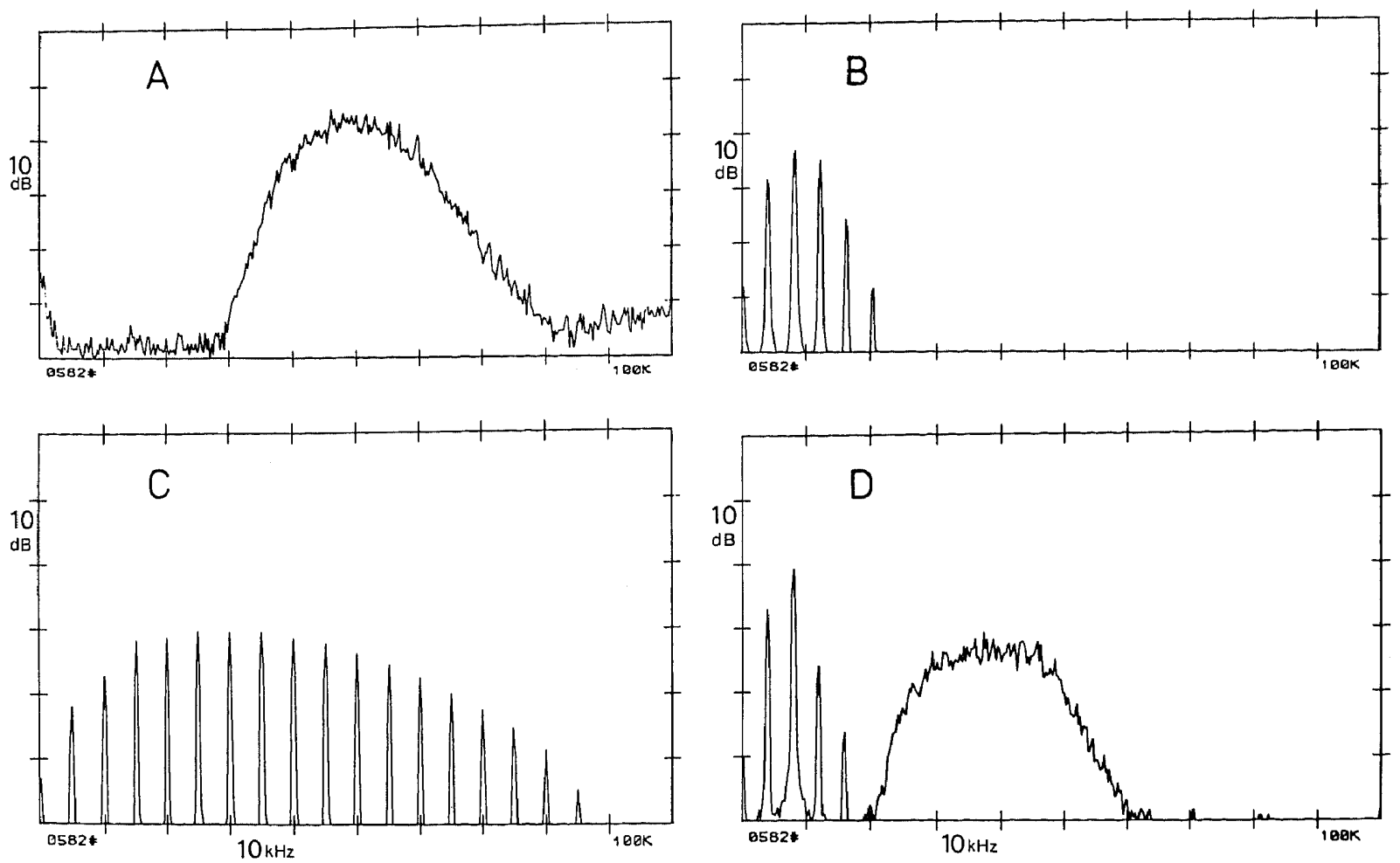

Fig. 1. Spectra of the four models of mouse calls presented as stimuli. Abscissa: frequency range $(0-100 \mathrm{kHz}$, with markers every $10 \mathrm{kHz}$ ); ordinate: relative $\mathrm{dB}$ (markers every $10 \mathrm{~dB}$ )

Table 1. Distributions of neurons from both anesthetic groups ( $\mathrm{N}$-group and T-group) into the response classes which we distinguished. Percentages are related to the total numbers of units in the $\mathrm{DC}+\mathrm{DM}$ or $\mathrm{CN}$ within each anesthetic group

\begin{tabular}{|c|c|c|c|c|}
\hline & \multicolumn{2}{|l|}{$\mathrm{N}$-group } & \multicolumn{2}{|l|}{ T-group } \\
\hline & $\mathrm{DC}+\mathrm{DM}$ & $\mathrm{CN}$ & $\mathrm{DC}+\mathrm{DM}$ & $\mathrm{CN}$ \\
\hline Total number of units & 25 & 76 & 15 & 48 \\
\hline Tone insensitive & 0 & $7(9 \%)$ & 0 & $1(2 \%)$ \\
\hline Tone preferred & $6(24 \%)$ & $11(14 \%)$ & $2(13 \%)$ & $6(12 \%)$ \\
\hline Call model insensitive & $8(32 \%)$ & $16(21 \%)$ & $3(20 \%)$ & $11(23 \%)$ \\
\hline Response to 1 call & $8(32 \%)$ & $8(10 \%)$ & $1(6 \%)$ & $4(8 \%)$ \\
\hline Response to 2 calls & $3(12 \%)$ & $11(14 \%)$ & $6(40 \%)$ & $14(29 \%)$ \\
\hline Response to 3 calls & $6(24 \%)$ & $32(42 \%)$ & $5(33 \%)$ & $18(37 \%)$ \\
\hline Response to 4 calls & 0 & $9(12 \%)$ & 0 & $1(1 \%)$ \\
\hline Noise insensitive & $13(52 \%)$ & $19(25 \%)$ & $8(53 \%)$ & $10(21 \%)$ \\
\hline Responsive to noise & $12(48 \%)$ & $57(75 \%)$ & $7(47 \%)$ & $38(79 \%)$ \\
\hline $\begin{array}{l}\text { Noise response but no call } \\
\text { response }\end{array}$ & $2(8 \%)$ & $5(6 \%)$ & $1(6 \%)$ & $5(10 \%)$ \\
\hline Call response but no noise response & $7(28 \%)$ & $8(10 \%)$ & $6(40 \%)$ & $5(10 \%)$ \\
\hline Noise preferred & 0 & $1(1 \%)$ & 0 & 0 \\
\hline
\end{tabular}

(tone preferred) or to broadband noise (noise preferred; only one unit in the N-group). As neurons which responded either to broadband noise or to one or several call models were seen, it is evident that a response to broadband noise did not necessarily indicate that the neuron would respond to a call model with frequency components within the noise bandwidth. It was also clear that units can respond very well to tones but not at all to broadband noise or to any call model even though the spectra of the broadband stimuli at least partially overlapped the tone-excitatory response area. Within the intensity range tested, neurons could exhibit a selective excitatory response only to one 
call (besides responses to tones and/or noise) or could, less selectively, respond to any combination or to all four call models as well as to tone and noise bursts. Thus we found, for different IC neurons, various degrees of neural response selectivity which could not be predicted by knowledge of the CFs of these units alone.

There were significant differences between the response probabilities to call models in the $\mathrm{DC}+$ $D M$ compared with the $\mathrm{CN}$ in the $\mathrm{N}$-group $(P<$ $0.05, \chi^{2}$-test). In the $\mathrm{CN}$, units generally responded to more calls than in the DC+DM. On the other hand, units in the DC+DM showed a higher probability for noise insensitivity and for call responses without noise response $(P<0.01$ and $P<0.05$ respectively, $\chi^{2}$-test). In the T-group, noise insensitivity and call responses without noise responses were also more frequently seen in the $\mathrm{DC}+\mathrm{DM}$ compared with the $\mathrm{CN} \quad(P<0.05$ and $P<0.01$, $\chi^{2}$-test). However, the difference between the distributions of call model responses did not occur (compare Table 1).

Neurons responding or not responding to the four call models have been evaluated separately (Fig. 2). Units with CFs within the spectrum of the respective call model might be expected to show an excitatory response to that call. Non-responding units, therefore, were neurons in which these expected responses did not occur. All units that might be expected to respond to the noise model of mouse pup ultrasounds (model A) did, in fact, respond. In the N-group, almost half of the units expected to respond to model B (lowfrequency harmonic call) did so; in the T-group all expected units responded to the $\mathrm{B}$ call. This is a significant difference $\left(P<0.01, \chi^{2}\right.$-test), presumably due to anesthetic effects. Neurons responded less frequently to the two-component call (model D) in the $\mathrm{N}$-group than in the T-group $\left(P<0.05, \chi^{2}\right.$-test). Units of the $\mathrm{N}$-group mice responded less frequently to call models to which a response might be expected. This significant difference between both anesthetic groups was based exclusively on units from the DC+DM (Fig. 2) which in the $\mathrm{N}$-group were less responsive to call models. No such differences existed for units of the $\mathrm{CN}$.

\section{Response thresholds}

Responding units had differences in threshold sensitivities to presented stimuli. Figure 3 shows thresholds to tone and noise bursts separately for the $\mathrm{N}$ - and T-group. Although the most sensitive units in both anesthetic groups had similar res-

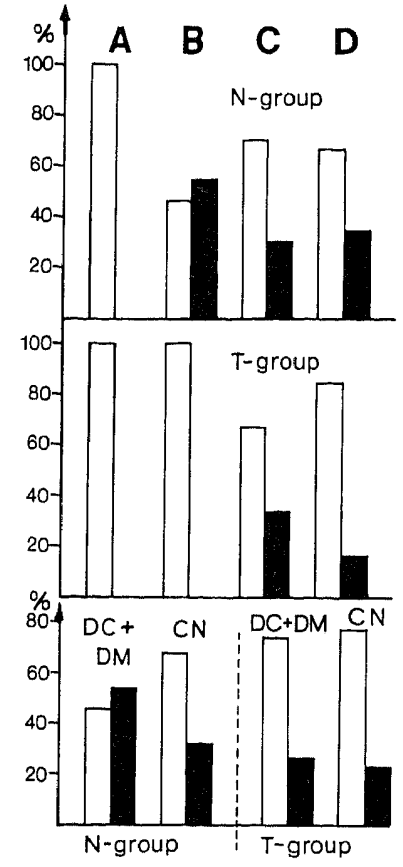

Fig. 2. Percentages of units responding (open bars) or nonresponding (black bars) to each of the four call models (separately for the $\mathrm{N}$ - and the T-group) and to all call models (separately for the $\mathrm{DC}+\mathrm{DM}$ and $\mathrm{CN}$ in each anesthetic group)

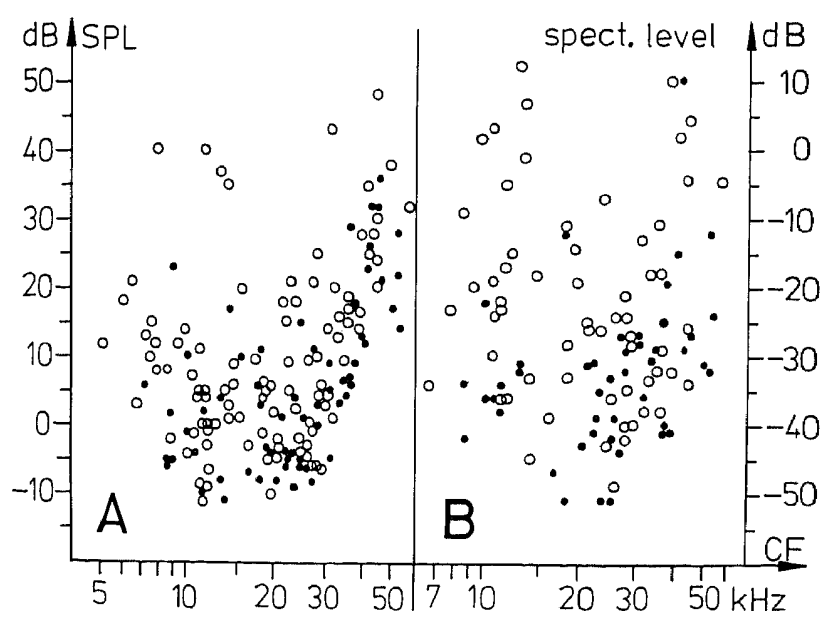

Fig. 3 A, B. Tone response (A) and noise response (B) thresholds of the units of the $\mathrm{N}$-group (open circles) and T-group (closed circles) as a function of their CFs

ponse thresholds, there were more rather insensitive neurons in the N-group, especially in response to noise. Response thresholds to the four call models as a function of CF are shown in Fig. 4. Nonresponding units are also indicated. In general, $\mathrm{N}$ and T-group units had comparable thresholds to all four calls. It is not surprising that lowest thresholds were found in units with CFs in the respective sound spectra. However, a considerable number 


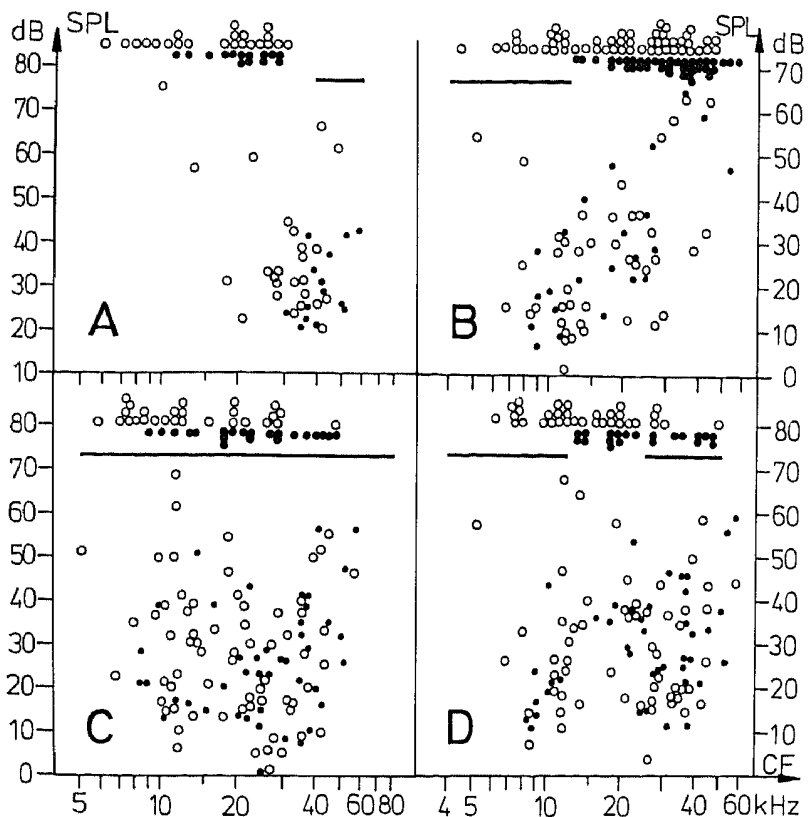

Fig. 4A-D. Response thresholds to the four call models (A-D) of units of the $\mathrm{N}$-group (open circles) and T-group (closed circles) as a function of their CFs. Non-responding units are also shown at the tops of the sub-figures. Frequency ranges of the call spectra are indicated by fat horizontal lines at the tops of the sub-figures

of neurons with CFs off the frequency ranges of the sound spectra responded although often with rather high thresholds. Thus, for example, the lowfrequency call B played at an intensity of $70 \mathrm{~dB}$ stimulated some neurons with CFs near $50 \mathrm{kHz}$ which is close to the upper frequency range of $\mathrm{CFs}$ occurring in this mouse strain (Ehret and Moffat 1985).

The differences between tone or noise thresholds and call response thresholds (tone minus call threshold or noise minus call threshold) can be used as a simple relative measure of stimulus preference. Such threshold differences are summarized in Fig. 5 for the two anesthetic groups. The occurrence of positive differences for all call models both in comparison with tone and noise thresholds illustrates that a certain percentage of units were more sensitive to a call model than to either $\mathrm{CF}$ tones or broadband noise. Average threshold differences were similar for all call models. When average Nand T-group values were compared, threshold differences from tone and call models did not differ significantly, whereas differences from noise and call model thresholds were significantly different $(P<0.02$ at least, $U$-test $)$. This is in accordance with Figs. 3 and 4 which showed already that the noise response thresholds of $\mathrm{N}$-group units and
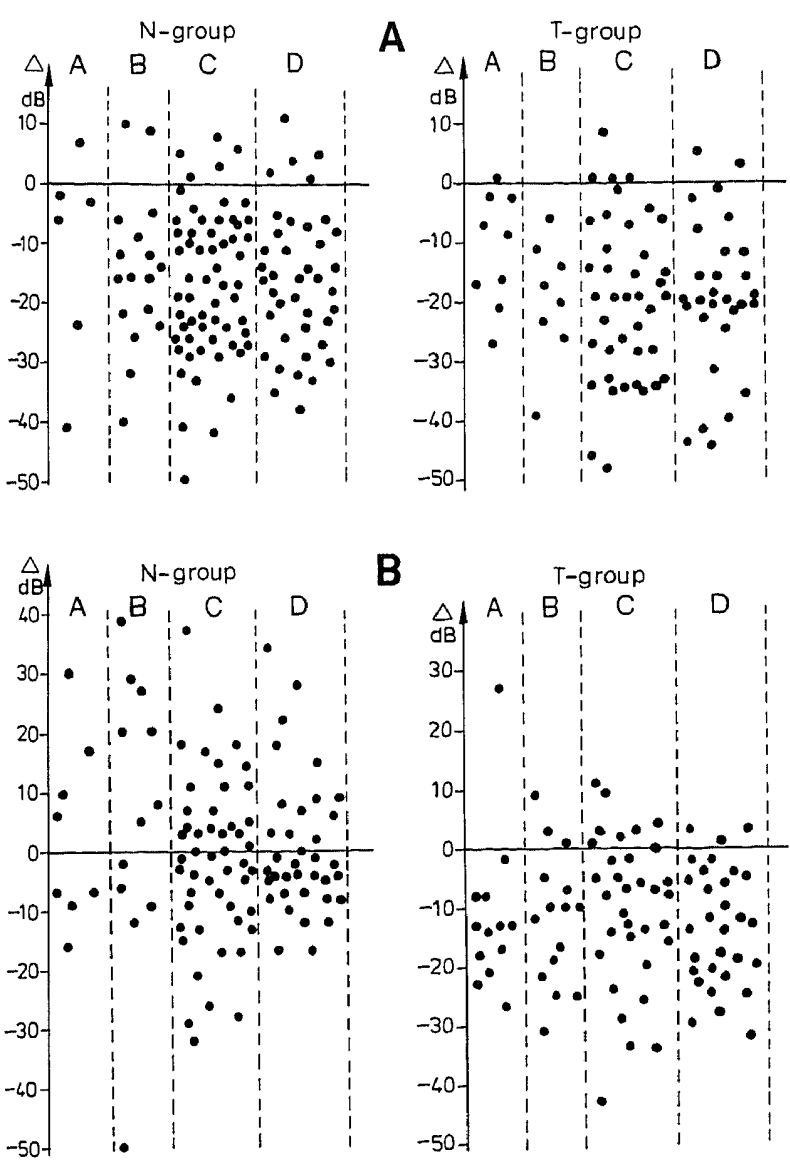

Fig. 5A, B. Tone response thresholds minus call response thresholds (A) and noise response thresholds minus call response thresholds (B) plotted separately for all four call models and for units from the $\mathrm{N}$ - and T-group

not the thresholds to the other stimuli were higher than those of the T-group. DC+DM and CN neurons did not differ significantly in this respect.

\section{Call responses and excitatory tuning}

In a previous paper (Ehret and Moffat 1985), we have divided excitatory tuning curves of IC units of the mouse into 7 classes according to their shapes. Examples of tuning curves of 6 classes are shown in Fig. 6. The average $Q_{10 \mathrm{~dB}}$-values (CF divided by the bandwidth of the tuning curve $10 \mathrm{~dB}$ above threshold) of the units in our sample were: class A: 8.9 ; class $\mathrm{B}: 2.0$; class $\mathrm{C}: 9.7$; class $\mathrm{D}$ : 23.0; class E: 6.1 and class $F: 6.4$. Class B units were significantly less sharply tuned compared with units of all other classes $(P<0.01, U$-test); class $\mathrm{E}$ units were less sharply tuned than class A $(P<0.02)$, class $\mathrm{C}(P<0.05)$ and class $\mathrm{D}(P<$ $0.05)$ units, and class $F$ units were less sharply 


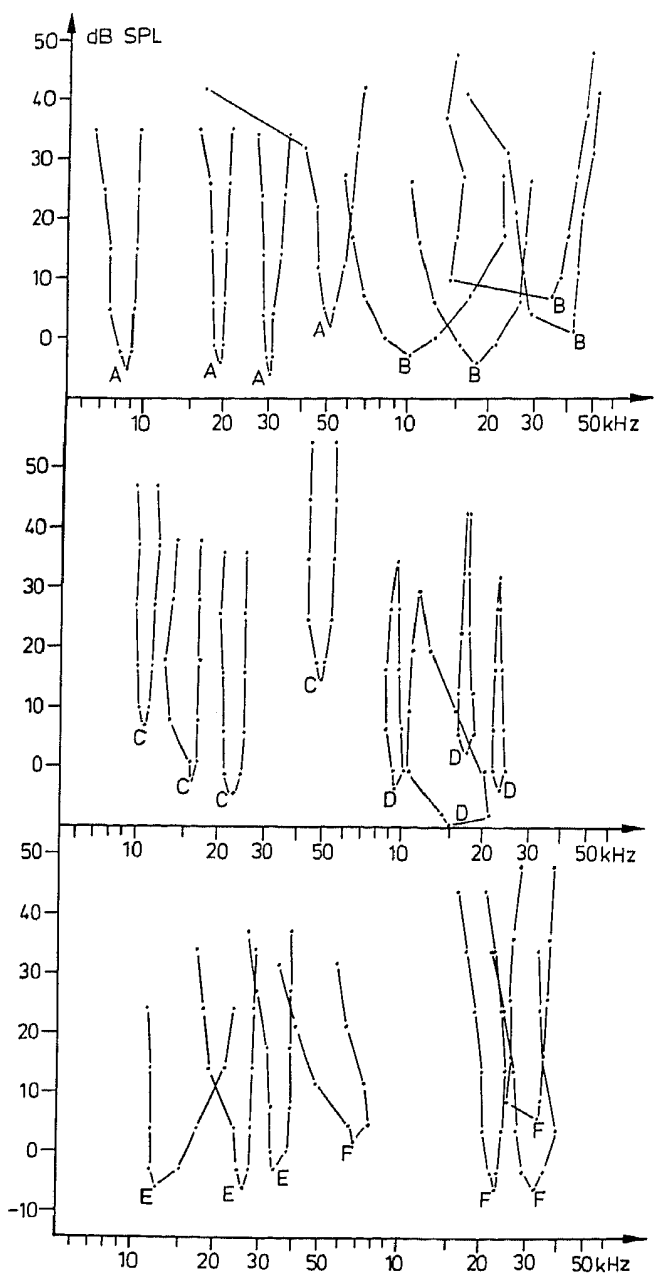

Fig. 6. Examples of excitatory tuning curves from the six different classes $(A-F)$

tuned compared with those in class A $(P<0.05)$. Figure 7 shows a plot of $Q_{10 \mathrm{~dB}}$-values vs. call response selectivity. A significant correlation was found between increasing selectivity and increasing $Q_{10 \mathrm{~dB}}$, i.e. sharpness of excitatory tuning (correlation coefficient $r=0.453 ; P<0.001$ ).

Table 2 shows clearly that more units with class $\mathrm{B}$ (broad tuning) but less with classes $\mathrm{A}$ and $\mathrm{C}$ (sharp tuning) tuning curve shapes responded to 3 and 4 call models compared to two, one or no call $\left(P<0.02\right.$ at least, $\chi^{2}$-test $)$. In addition, higher proportions of class $A$ and $C$ units are noise insensitive and tone preferred compared with neurons from classes B, E and F. This shows that the shapes of excitatory tuning curves and their sharpness (expressed by the $Q_{10 \mathrm{~dB}}$-value) are meaningful but not absolute indicators for the degree of specialization of neurons in the IC to respond to complex sound spectra. All tuning curve classes were found

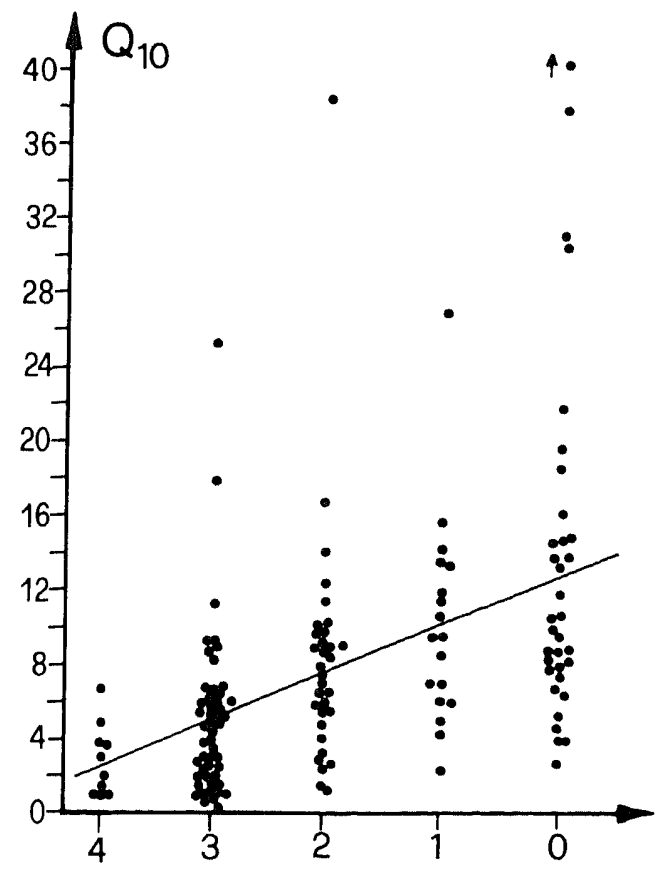

Fig. 7. $Q_{10 \mathrm{~dB}}$-values of the tuning curves plotted against the degree of selectivity of responses to the mouse call models. $0=$ units which did not respond to any call model; $1,2,3$, $4=$ units responding to $1,2,3$, or 4 call models, respectively

Table 2. Distribution of neurons with different response selectivity into the six tuning curve classes (A-F). Data from all neurons pooled

\begin{tabular}{lrrrrrrr}
\hline & A & B & C & D & E & F & $\Sigma$ \\
\hline Tone preferred & 6 & 0 & 11 & 4 & 3 & 1 & 25 \\
Noise insensitive & 15 & 3 & 18 & 6 & 5 & 3 & 50 \\
Call model insensitive & 12 & 0 & 12 & 6 & 4 & 4 & 38 \\
Response to 1 call & 8 & 0 & 9 & 2 & 2 & 0 & 21 \\
Response to 2 calls & 16 & 3 & 8 & 0 & 3 & 4 & 34 \\
Response to 3 calls & 17 & 22 & 6 & 4 & 7 & 5 & 61 \\
Response to 4 calls & 0 & 6 & 0 & 0 & 4 & 0 & 10 \\
\hline
\end{tabular}

in both anesthetic states and in the DC+DM and the CN (compare Ehret and Moffat 1985).

\section{Discussion}

Within the intensity range of up to $80 \mathrm{~dB}$ SPL of the stimuli used in the present study various degrees of selectivity of inferior colliculus units for 4 mouse call models and noise were seen. The predictability of an excitatory response to a complex sound stimulus is $100 \%$ in the auditory nerve. It is clearly less than that in the IC. This is true both for the two nucleus regions we distinguished, and for the anesthetic protocols used. Of all units ex- 
pected to respond to broadband noise because of their $\mathrm{CF}$ being within the noise band only $75-79 \%$ of $\mathrm{CN}$ neurons and $47-48 \%$ of DC+DM neurons actually responded. Expected responses to call models occurred in $70-80 \%$ of the neurons. Suga (1969) and Engelstätter et al. (1980) also found noise insensitive neurons in the IC of bats (presumably in the $\mathrm{CN}$ ), however in much lower percentages $(1-19 \%)$. Reasons for these discrepancies could be, besides species differences, the shorter stimulus durations for the noise and the smaller bandwidths used in these studies. Scheich et al. (1977) studied the responses of units in the nucleus mesencephalis dorsalis lateralis (corresponding to the IC) of the Guinea fowl to variants of three intraspecific calls. They found $75 \%$ of the units responding to calls, which closely corresponds to the $70-80 \%$ we found in the present study for the mouse.

In the auditory cortex of unanesthetized squirrel monkeys $41 \%$ or $42 \%$ of studied units responded to monkey calls or noise bursts respectively (Funkenstein and Winter 1973). This is a greater selectivity than in the neuronal population studied in the mouse IC. Because sound intensity was constant at $70 \mathrm{~dB}$ SPL in the monkey study, most probably a higher proportion of responsive units would have been found if an intensity range of up to $80 \mathrm{~dB}$ SPL had been covered, as in the present investigation. Other single unit data of the AI of the auditory cortex of unanesthetized cats (Goldstein et al. 1968) revealed about the same proportion of units responsive to noise bursts $(74 \%)$ as has been found in the $\mathrm{CN}$ of the IC of the mouse. The study of Goldstein et al. (1968) was restricted to the AI, whereas Funkenstein and Winter (1973) probably also recorded from neurons within other auditory fields, which might explain these differences.

It is important to note that, in the T-group, neurons with CFs within the spectra of the two narrowband calls (above $40 \mathrm{kHz}$ for model $\mathrm{A}$ and below $12 \mathrm{kHz}$ for model $\mathrm{B}$ ) all responded to the respective call models (Figs. 2, 4). The same units and those with CFs between 12 and $40 \mathrm{kHz}$ did not necessarily respond to the wideband calls (models C and D) and to broadband noise. Thus calls with sound energy concentrated near the upper or lower end of the audible frequency range of the mouse only, are processed in the IC in a more predictable way with regard to our criterion (response or no response). The temporal patterns of the single unit responses to the calls can, of course, be very variable (Ehret and Moffat 1985). The reason for the higher responsiveness in the
Table 3. Distribution of neurons with different characteristic frequencies $(\mathrm{CF}$ ) into tuning curve classes $\mathrm{A}+\mathrm{B}$ (primary-like and broad) and $C+D+E+F$ (indicating inhibitory side bands). Data from all neurons pooled. $P=$ significance level

\begin{tabular}{llcl}
\hline CF & A +B & C +D E E F & $P$ \\
\hline$<10 \mathrm{kHz}$ & 10 & 0 & $<0.01$ \\
$10-20 \mathrm{kHz}$ & 20 & 36 & $<0.05$ \\
$20-30 \mathrm{kHz}$ & 14 & 29 & $<0.05$ \\
$30-40 \mathrm{kHz}$ & 21 & 12 & $\mathrm{~ns}$ \\
$>40 \mathrm{kHz}$ & 19 & 3 & $<0.01$ \\
\hline
\end{tabular}

IC for low and high frequency calls might have to do with the strength of inhibitory influences on the excitatory responses. Although the shapes of the inhibitory surrounds of excitatory tuning curves and the strength of inhibition have not been studied systematically as a function of $\mathrm{CF}$, it is conceivable that units with rather low $(<10 \mathrm{kHz})$ or high $(>40 \mathrm{kHz}) \mathrm{CFs}$ lack extensive or strong inhibitory side bands at the low or high frequency slope of their excitatory tuning curves. By that, excitation can be expected to dominate the response to a rather narrowband sound spectrum. Units with CFs in the middle frequency range of hearing are more likely to have a strong inhibitory surround of their excitatory frequency area and therefore could be totally inhibited by a broad sound spectrum. This suggestion is supported by the distribution of excitatory tuning curve shapes into CF classes (Table 3). Units with CFs below 10 or above $40 \mathrm{kHz}$ have significantly more often $\left(P<0.01, \chi^{2}\right.$-test) primary-like (class A) or broad (class B) tuning curves than other shapes (classes $\mathrm{C}-\mathrm{F}$ ), which indicate inhibitory side bands by having very steep slopes or being closed or tilted. Midfrequency units have significantly more frequently the shapes C-F (Fig. 6). Thus our data indicate a relationship between higher order tuning curve shapes with supposedly inhibitory side bands and higher response selectivity to complex sound spectra.

In both anesthetic groups a significantly higher proportion of neurons in the DC+DM compared to the $C N$ were noise insensitive and showed a call but no noise response (Table 1). The reason for this difference could be related to the small bandwidths of spectral integration (critical bands; mostly smaller than $0.5 \mathrm{kHz}$ below $10 \mathrm{kHz} \mathrm{CF}$; Ehret to be published). With these narrow bandwidths only a small amount of spectral noise energy may become effective in stimulating an excitatory response in a unit. Since broadband noise does also stimulate inhibitory side bands (if present), 
inhibitory effects to a noise stimulus may dominate in some units and thus block an excitatory response. Responses to harmonically structured calls should be less affected because the energy concentrated at harmonics is easily integrated within a critical bandwidth.

Of the units that responded to noise bursts, response thresholds in the $\mathrm{N}$-group were considerably higher compared with those of T-group units (Figs. 3, 5). This threshold increase could be due to the depressive effects that barbiturates have on the central nervous system (Westhues and Fritsch 1961). In the auditory system from the dorsal cochlear nucleus upwards sodium pentobarbital is known to (a) reduce spontaneous activity in general (Bock et al. 1972; Webster and Aitkin 1975), (b) reduce lateral inhibition in the dorsal cochlear nucleus (Evans and Nelson 1973), (c) increase presynaptic inhibition in the IC (Nelson and Erulkar 1963; Watanabe and Simada 1970, see also Ryan and Miller 1978). A reduction of excitation or excitability in the IC can be expected to increase response thresholds especially to broadband noise because of the spectral integration necessary to reach the threshold.

A further barbiturate effect in the present study was the decrease of responsiveness to the call models in the DC+DM compared with the CN and with the T-group (Fig. 2). This result is difficult to interpret because it indicates an influence of Nembutal specifically on the DC $+D M$. Since the present data showed already that $\mathrm{DC}+\mathrm{DM}$ units were less responsive to noise and Nembutal increased response thresholds especially to noise, one could assume that if both factors had added together, DC+DM neurons became also less responsive to other complex signals like animal call models.

Acknowledgements. This study was supported by the Deutsche Forschungsgemeinschaft, Eh 53/4, 5, 6, 8. We thank Drs. M.M. Merzenich and G. Langner for helpful comments on the manuscript.

\section{References}

Bock GR, Webster WR, Aitkin LM (1972) Discharge patterns of single units in the inferior colliculus of the alert cat J Neurophysiol 35:355-372

Dräger UC (1975) Receptive fields of single calls and topography in mouse visual cortex. J Comp Neurol 160:269-290

Ehret G (1975) Schallsignale der Hausmaus, Mus musculus. Behaviour 52:38-56

Ehret G, Haack B (1982) Ultrasound recognition in house mice: key-stimulus configuration and recognition mechanism. J Comp Physiol 148:245-251

Ehret G, Moffat AJM (1984) Noise masking of tone respontses and critical ratios in single units of the mouse cochlear nerve and cochlear nucleus. Hearing Res 14:45-57

Ehret G, Moffat AJM (1985) Inferior colliculus of the house mouse II: single unit responses to tones, noise, and tonenoise combinations as a function of sound intensity. J Comp Physiol A 156:619-635

Engelstätter R, Vater M, Neuweiler G (1980) Processing of noise by single units of the inferior colliculus of the bat Rhinolophus ferrumequinum. Hearing Res 3:285-300

Evans EF, Nelson PG (1973) The responses of single neurons in the cochlear nucleus of the cat as a function of their location and the anaesthetic state. Exp Brain Res $17: 402-427$

Funkenstein HH, Winter P (1973) Responses to acoustic stimuli of units in the auditory cortex of awake squirrel monkeys. Exp Brain Res 18:464-488

Goldstein MH, Hall JL, Butterfield BO (1968) Single-unit activity in the primary auditory cortex of unanesthetized cats. J Acoust Soc Am 43:444-455

Kiang NYS, Moxon EC (1974) Tails of tuning curves of auditory nerve fibers. J Acoust Soc Am 55:620-630

Langner G, Bonke D, Scheich H (1981) Neuronal discriminition of natural and synthetic vowels in field $L$ of trained mynah birds. Exp Brain Res 43:11-24

Machmerth H, Theiss D, Schnitzler HU (1975) Konstruktion eines Luftschallgebers mit konstantem Frequenzgang im Bereich von $15 \mathrm{kHz}$ bis $130 \mathrm{kHz}$. Acustica 34:81-85

Møller AR (1970) Unit responses in the cochlear nucleus of the rat to noise and tones. Acta Physiol Scand 78:289-298

Newman JD (1979) Central nervous system processing of sounds in primates. In: Steklis HD, Raleigh MJ (eds) Neurobiology of social communication in primates: an evolutionary perspective. Academic Press, New York, pp 69-109

Rhode WS, Geisler CD, Kennedy DT (1978) Auditory nerve fiber responses to wide-band noise and tone combinations. J Neurophysiol $41: 692-704$

Ruggero MA (1973) Response to noise of auditory nerve fibers in the squirrel monkey. J Neurophysiol 36:569-587

Ryan A, Miller J (1978) Single unit responses in the inferior colliculus of the awake and performing rhesus monkey. Exp Brain Res 32:389-407

Scheich H, Langner G, Koch R (1977) Coding of narrow-band and wide-band vocalizations in the auditory midbrain nucleus (MLD) of the Guinea fowl (Numida meleagris). J Comp Physiol 117:245-265

Suga N (1969) Classification of inferior colliculus neurons of bats in terms of responses to pure tones, FM sounds and noise bursts. J Physiol 200:555-574

Symmes D, Alexander GE, Newman JD (1980) Neural processing of vocalizations and artificial stimuli in the medial geniculate body of squirrel monkeys. Hearing Res 3:133-146

Watanabe T, Simada Z (1970) Collicular auditory interneuron and its blocking by dihydro- $\beta$-erythroidene hydrobromide. Proc Jpn Acad 46:983-988

Webster WR, Aitkin LM (1975) Central auditory processing. In: Gazzanig MS, Blakemore C (eds) Handbook of psychobiology. Academic Press, New York, pp 325-364

Westhues M, Fritsch R (1961) Die Narkose der Tiere, Bd II. Allgemeinnarkose. Parey, Berlin

Young ED, Brownell WE (1976) Responses to tones and noise of single cells in dorsal cochlear nucleus of unanesthetized cats. J Neurophysiol 39:282-300 